\title{
Soziale Arbeit braucht qualifizierte Grundlagenforschung
}

\section{Die Stichworte lauten Finanzierung, Forscherpersönlichkeiten, Interdisziplinarität}

Konrad Maier

Prof. Dr. Konrad Maier war bis seiner Emeritierung Hochschullehrer für Politikwissenschaft und Sozialarbeitswissenschaft an der Evangelischen Fachhochschule Freiburg sowie Gründer und langjähriger Leiter des Forschungsinstituts dieser Fachhochschule.

E-Mailmaier@efh-freiburg.de
Die Soziale Arbeit hat in den letzten drei Jahrzehnten stark expandiert. Mit dieser Expansion hat die Entwicklung der Wissenschaft der Sozialen Arbeit kaum Schritt halten können. Gebraucht wird vor allem eine eigenständige Grundlagenforschung, die jedoch beträchtliche personelle und finanzielle Ressourcen erfordert.

Die Verberuflichung Sozialer Arbeit ist von Anfang an eng mit empirischer Forschung verbunden. In den USA war die Begründung der praktischen Sozialarbeit in Hull House unmittelbar eingebettet in die universitäre Sozialforschung und die Begründung der »Chicagoer Schule « der Sozialwissenschaft und - wenn man so will - der Sozialarbeitswissenschaft. In Deutschland führten große Sozialenqueten über das Pflegekinderwesen zu sozialen Reformen im wilhelminischen Reich und zur Verberuflichung der Sozialen Arbeit.

Während in den USA und in zahlreichen anderen Ländern die Ausbildung in Social Work an den Hochschulen angesiedelt und damit eine wissenschaftliche Disziplin "Social Work « und eine entsprechende Forschung begründet wurde, hat sich in Deutschland bis zum letzten Quartal des 20. Jahrhunderts keine entsprechende Sozialarbeitsforschung entwickelt. Dies ist einmal darin begründet, dass das Thema Soziale Arbeit an den deutschen Universitäten von geisteswissenschaftlich orientierten Pädagogen aufgegriffen wurde, die zwar vielfältige philosophisch/pädagogische Theorien und Konzepte entwickelten, denen aber empirische Forschung fernlag. Zum anderen entwickelte sich die Ausbildung der Fürsorgerinnen ganz überwiegend an sozialen Frauenschulen, in denen von der Anlage her empirische Forschung nur sehr begrenzt - als Freizeithobby engagierter Dozentinnen - möglich war.

\section{Take-off der Sozialarbeitsforschung}

Dies änderte sich grundlegend, zum einen damit, dass sich Pädagogik - und auch Sozialpädagogik - zur Erziehungswissenschaft weiterentwickelte und damit empirische Forschung zu einem Essential des wissenschaftlichen Arbeitens wurde, zum andern mit der Verlagerung der Ausbildung von Sozialarbeiterinnen und Sozialarbeitern sowie Sozialpädagoginnen und Sozialpädagogen auf die Fachhochschulen.

Hans-Uwe Otto konstatiert Anfang des 21. Jahrhunderts eine »Take-offPhase empirischer Forschung in dem kombinierten Bereich Sozialarbeit/Sozialpädagogik «. Dieselbe Feststellung traf Piotr Salustowicz bereits Ende der 1980er Jahre vor dem Hintergrund einer Untersuchung der Forschung an den Fachhochschulstudiengängen Sozialarbeit/Sozialpädagogik in Nordrhein-Westfalen. Inzwischen haben fast alle Hochschulstudiengänge Soziale Arbeit einen Forschungsbeauftragten, zwei Drittel verfügen über ein eigenes Forschungsinstitut, jeder dritte Hochschullehrende ist in der Forschung engagiert. Im Jahre 2006 waren im rechnerischen Durchschnitt der befragten Studiengänge 3,6 hauptamtliche wissenschaftliche Mitarbeiterinnen und Mitarbeiter beschäftigt, hinzukommen rechnerisch 3,3 studentische Hilfskräfte. Zwischen den Fachhochschulen gibt es jedoch beträchtliche Unterschiede. So waren Ende 2007 im Forschungsinstitut der Evangelischen Fachhochschule Freiburg 36 hauptamtliche Mitarbeiterinnen und Mitarbeiter (auf 27 Vollzeitstellen) beschäftigt. Die Bedeutung der Forschung für das Klima an dieser Hochschule wird insbesondere darin deutlich, dass in Freiburg jeder zehnte Studierende zeitweise als studentische Hilfskraft bei Forschungsprojekten mitarbeitet. 
In den letzten 20 Jahren sind die meisten Forschungsprojekte im Bereich der Sozialen Arbeit den beiden klassischen Formen der Sozialarbeitsforschung zuzuordnen: der Analyse von sozialen Verhältnissen und sozialen Problemlagen sowie der Evaluation Sozialer Arbeit. In einer Mischform zwischen diesen beiden Forschungstypen werden Untersuchungen angestellt zur Planung neuer sozialer Dienste oder auch zur längerfristigen wissenschaftlichen Begleitung innovativer Projekte, wie sie häufig von den Geldgebern (z. B. dem Europäischen Sozial- sem forschenden Lernen teilhaben lassen. Die Praxis geht nicht mehr als persönliche Erfahrung früherer Praxis in die Lehre ein, sondern als wissenschaftlich bereits reflektierte Erfahrung der aktuellen Praxis.

In diesem Sinne hat praxisorientierte Forschung eine kaum zu überschätzende Bedeutung für die Entwicklung der Praxis Sozialer Arbeit wie auch für die Lehre an den Fachhochschulen. Der Beitrag dieser Forschung zur Weiterentwicklung von Theorie ist jedoch begrenzt. Für die Annahme, dass bei einer Zunahme dieser

»Forschung hat eine kaum zu überschätzende Bedeutung für die Entwicklung der Praxis Sozialer Arbeit wie auch für die Lehre an den Fachbochschulen «

fonds) vorgesehen sind. Diese Untersuchungen werden teilweise als Unterrichtsprojekte ohne oder mit geringfügigen finanziellen Mitteln durchgeführt, zunehmend sind jedoch örtliche und regionale Träger bereit, für derartige Forschungen auch eine entsprechende Finanzierung sicher zu stellen. Zugleich lässt sich feststellen, dass kommunale, staatliche und freie Träger Forschungsaufträge nicht mehr ausschließlich oder primär an universitäre Institute vergeben, sondern angesichts der erkennbaren Leistung der Sozialarbeitsforschung kleinere Forschungsaufträge zunehmend an Fachhochschulinstitute vergeben.

Diese praxisorientierte Forschung trägt erheblich dazu bei, dass im regionalen Umfeld der Fachhochschulen die Praxis mit wissenschaftlicher Theorie konfrontiert wird und dass bestehende Praxis reflektiert und korrigiert wird und innovative Projekte gefördert werden. Zugleich verändert diese Forschung grundlegend die Lehre: die Lehrenden sind nicht mehr primär "Meister ", die ihre früher erworbenen Kenntnisse und Erfahrungen (als »lehrende Sozialarbeiterinnen und Sozialarbeiter «) weitergeben, sondern Wissenschaftlerinnen und Wissenschaftler, die permanent neu lernen und andere an die-
Art von praxisorientierter Forschung die Quantität in eine Qualität umschlägt in dem Sinne, dass die große Zahl von Einzelforschungen fundierte theoretische Aussagen ermöglicht, gibt es wenige konkrete Anzeichen. Die Projektdarstellungen in den verschiedenen Dokumentationen lassen deutlich erkennen, dass der Erkenntnisgewinn für das je konkrete Projekt deutlich im Vordergrund steht und eher gezwungen ein Bezug zur Theorieentwicklung formuliert wird. Die Produzenten von Theorie setzen sich primär mit den gerade vorherrschenden großen Theoriesystemen auseinander, eine Vermittlung mit der alltäglichen Praxis und deren Forschung erscheint hier wenig hilfreich und außerdem ist die Praxis und eben auch deren empirische Erforschung durchaus sperrig.

Ein unmittelbarer Beitrag zur Theoriediskussion wird am ehesten in Diplomarbeiten und Dissertationen geleistet, wenn sie theoretisch entwickelte Konzepte auf ihre Tragfähigkeit hin überprüfen oder auf einige konkrete Situationen hin präzisieren. Dies kann jedoch eine Grundlagenforschung im Bereich Sozialer Arbeit nicht ersetzten. Deren Aufgaben können folgendermaßen beschrieben werden:

- Eine empirisch tragfähige Fundierung der Theorien der Sozialen Arbeit ist nur möglich, wenn das praktische Handeln, das vielfach intuitiv erfolgt und in hohem Maße auf »implizitem Wissen « fußt, entschlüsselt wird. Nur durch eine sorgfältige Erhebung der tatsächlichen Praxis gelingt es, das Handeln der Sozialarbeiterinnen und Sozialarbeiter $\mathrm{zu}$ beschreiben und damit einer kritischen Reflexion zuzuführen. Hierzu ist ein Mix von hochelaborierten Methoden empirischer Sozialforschung erforderlich.

- Die besondere Leistung der Sozialen Arbeit liegt - vermutlich - darin, in Kooperation mit Klientinnen und Klienten Lösungen für komplexe soziale Probleme zu entwickeln. Nur wenn die so gefundenen Lösungen dokumentiert und reflektiert werden, können sie in eine Fachöffentlichkeit wie auch in die Politik transportiert werden mit dem Ziel, die Rahmenbedingungen für die Lösung dieser Probleme zu verbessern.

- Unter dem Label »Integrierte Praxisforschung « wurde im Bereich der Sozialen Arbeit eine Form der Entwicklung von Verfahren aus der Ingenieurwissenschaft übernommen: Zur Lösung komplexer Handlungsprobleme arbeiten Wissenschaftler und Praktiker aus verschiedenen Berufszusammenhängen zeitlich begrenzet projektförmig zusammen mit dem doppelten Ziel, für eine konkrete Aufgabe der Praxis eine Lösung zu finden und zugleich ein verallgemeinerbares Verfahren zu entwickeln. Bei dieser hybriden Form der Wissensproduktion (»Modus 2« im Gegensatz zu den traditionellen Wissenstransfers von der Wissenschaft zur Praxis hin) wird das »implizite Wissen « und Kreativität der Praktikerinnen und Praktiker in die Entwicklung von Verfahren ebenso eingebracht wie das wissenschaftliche Wissen.

\section{Soziale Arbeit braucht eine eigene Forschungsförderung}

Eine derartige Forschung ist nur möglich unter Anwendung elaborierter Formen der Sozialforschung, sie erfordert beträchtliche personelle und finanzielle Ressourcen. Diese »Grundlagenforschung « liegt nicht im unmittelbaren Verwertungsinteresse von örtlichen oder regionalen Trägern der Sozialen Arbeit und 
sie übersteigt in der Regel auch deren finanzielle Möglichkeiten.

Der Zugang zu den großen Fördereinrichtungen für Grundlagenforschung (Deutsche Forschungsgemeinschaft, VW Stiftung, Thyssen Stiftung u. a.) ist für die Soziale Arbeit weithin verschlossen angesichts der Dominanz der Gutachter aus den klassischen Disziplinen. Ähnliches gilt für die »Ressort-Forschung «, für die Bund und Länder sowie zunehmend die Europäische Kommission beträchtliche Mittel zur Verfügung stellen. So sind die größeren und komplexen Forschungsprojekte im organisatorischen Rahmen der Studiengänge Soziale Arbeit überwiegend in Randgebieten der Sozialen Arbeit angesiedelt, wo es größere Schnittmengen gibt zur Gesundheitsforschung, zur Frauen- und Genderforschung, zur Pädagogik der Frühen Kindheit, zur Gerontologie, zur Rechtstatsachenforschung u. ä. In diesem Sinne hat beispielsweise der Spitzenverband der Pflegekassen dem Forschungsinstitut der Evangelischen Fachhochschule Freiburg fünf Millionen zur Verfügung gestellt zur Entwicklung und Erprobung neuer Formen der Leistungserbringung in Form eines Pflegebudgets unter Einbeziehung von Case-Managern.

Nach den bisherigen Erfahrungen erscheint für eine fundierte Grundlagenforschung im Bereich der Sozialen Arbeit ein eigener Forschungstopf erforderlich, wie er auf dem Würzburger Forschungskongress der Deutschen Gesellschaft für Sozi- ale Arbeit 2007 gefordert wurde (siehe Kasten unten). Immerhin werden im Gesundheitswesen fünf Prozent des Gesamtbudgets für Forschung ausgegeben, im Bereich der Sozialen Arbeit würde eine Summe in Höhe von einem halben Prozent der Ausgaben für Soziale Arbeit eine komfortable Forschung ermöglichen.

\section{Sozialarbeitsforschung braucht qualifizierte Forschungs- persönlichkeiten und Interdisziplinarität}

Neben Geld sind jedoch qualifizierte Forscherpersönlichkeiten eine unabdingbare Voraussetzung für gute Grundlagen-

\section{Für eine Stiftung, für ein Programm zur Förderung der Sozialarbeitsforschung}

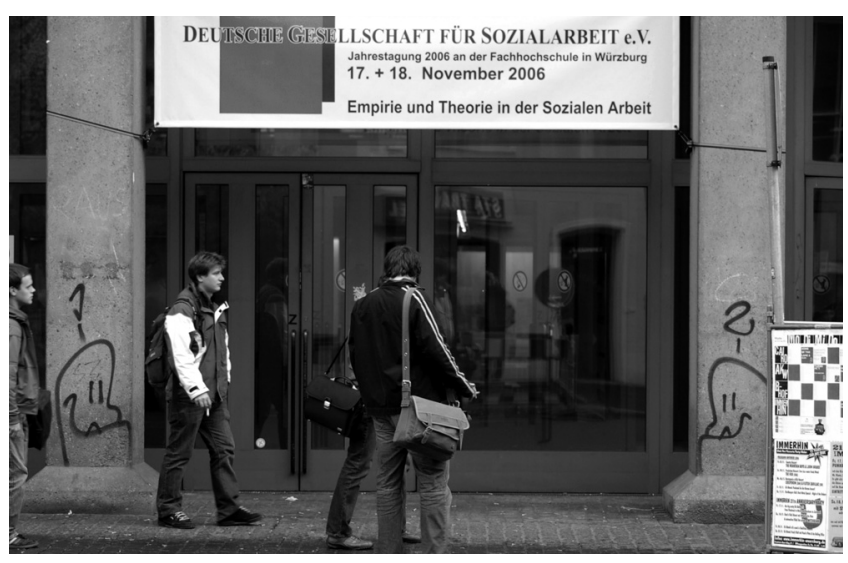

Der Jahreskongress der Deutschen Gesellschaft für Soziale Arbeit e. V. hat sich im Jahre 2006 in Würzburg mit Fragen der Forschung in der Sozialen Arbeit beschäftigt und dabei zahlreiche Projekte und Studien diskutiert. Verabschiedet wurde dabei auch ein von Prof. Dr. Konrad Maier formulierter Aufruf für eine gesicherte Finanzierung von Forschung im Feld der Sozialen Arbeit.

1 Die Soziale Arbeit verzeichnet in Deutschland in den letz1 - ten 30 Jahren eine beispiellose Expansion: Die Zahl der sozialversicherungspflichtig beschäftigten Diplom-SozialarbeiterInnen/Diplom-SozialpädagogInnen hat sich verfünffacht, auch in den letzten 10 Jahren ist diese Zahl trotz des allerorten festgestellten Sozialabbaus um $23 \%$ angestiegen. Offensichtlich nimmt die Zahl der Problemlagen, deren Lösung die Gesellschaft der Sozialen Arbeit zuschreibt, kontinuierlich zu.

2 Mit dieser Expansion hat die Entwicklung der Wissen• schaft und Forschung der Sozialen Arbeit nicht Schritt gehalten, im Vergleich mit anderen Disziplinen wie z. B. dem Ingenieurwesen liegt die wissenschaftliche Begründung in der Sozialen Arbeit für die Bearbeitung ihres Gegenstandsbereiches weit zurück.
3 Inzwischen wurden auch an den Fachhochschulen be3 merkenswerte Forschungskapazitäten im Bereich der Sozialen Arbeit aufgebaut; und die Praxis fragt zunehmend Evaluationsuntersuchungen, wissenschaftliche Begleitung, Feldanalysen und die Mitarbeit bei der Planung sozialer Dienste nach.

$\triangle$ Für eine immer wichtiger werdende Grundlagenfor• schung im Bereich Sozialer Arbeit fehlen jedoch die erforderlichen Ressourcen. Die Forschungsförderung ist sehr stark auf die traditionellen Disziplinen hin orientiert, die Gutachter rekrutieren sich fast ausschließlich aus traditionellen universitären Disziplinen und zeigen wenig Verständnis für die sozialarbeiterischen Fragen und wenig Bereitschaft, den kleiner werdenden Kuchen mit Newcomern zu teilen. Wir beobachten, dass Grundlagenforschung im Bereich Sozialer Arbeit am ehesten finanziert wird, wenn sich Überschneidungen mit klassischen Disziplinen und großen gesellschaftlichen Problemen ergeben, z. B. im Bereich des Gesundheitswesens, der Gerontologie, der Erziehung der frühen Kindheit, der Pflege, des Gender Mainstreamings. Die Entwicklung einer eigenständigen Sozialarbeitswissenschaft und einer ihr angemessen Forschung wird auf diese Weise behindert.

5 Wir fordern die großen Träger der Sozialen Arbeit, Städ$\mathcal{S}$ •te und Landkreise, Wohlfahrtsverbände und die einschlägigen Ministerien in Bund und Ländern auf, eine eigene Stiftung oder ein eigenes Förderprogramm einzurichten für die Förderung von Forschung im Bereich Sozialer Arbeit. Wenn nur ein Betrag in der Höhe eines kleinen Bruchteils (z. B. 0,5 Prozent) der Ausgaben für Soziale Arbeit in die Förderung von einschlägiger Forschung investiert wird, kann eine der Sozialen Arbeit angemessene Forschung ermöglicht werden.

6 Die Vergabepraxis wie auch die Ansprüche an die Qua•lität der Forschung sollte sich durchaus an der bestehenden Forschungsförderung wie z. B. der Deutschen Forschungsgemeinschaft orientieren, sich jedoch ganz auf die Fragestellungen im Bereich der Sozialen Arbeit konzentrieren. 
forschung. Die größeren und komplexeren Forschungsprojekte in Sozialer Arbeit sind - fast - durchgängig initiiert und geleitet von Vertretern der "Bezugswissenschaften «, während sich die gelernten Sozialarbeiterinnen und Sozialarbeiter stärker der Praxisberatung und kleineren Unterrichtsprojekten widmen. Dies ist durchaus verständlich, da die Sozialarbeiterinnen und Sozialpädagogen ein praxisorientiertes Studium absolviert haben und in der Regel ohne den Rückhalt eines wissenschaftlichen Instituts promoviert und vielfach noch nebenher praktische Sozialarbeit geleistet haben. Dem gegenüber haben die Soziologen, Psychologen und Politologen in der Regel im Umkreis eines wissenschaftlichen Instituts promoviert und vielfach als Assistentinnen und

\section{Literatur}

Forschung für die Praxis, hg. von Ernst Engelke/Konrad Maier/Erika Steinert/ Stefan Borrmann/Christian Spatscheck, Freiburg im Breisgau 2007.

Thomas Klie/Paul Stefan Roß (Hg.): Sozialarbeitswissenschaft und angewandte Forschung in der Sozialen Arbeit. Festschrift für Konrad Maier, Freiburg im Breisgau 2007.

Cornelia Schweppe/Werner Thole (Hg.): Sozialpädagogik als forschende Disziplin, Weinheim 2005.

Hans-Uwe Otto/Gertrud Oelerich/ Heinz-Günther Micheel (Hg.): Empirische Forschung und Soziale Arbeit, München/Unterschleißheim 2003.

Konrad Maier (Hg.): Forschung an Fachhochschulen für Soziale Arbeit. Bestandsaufnahme und Perspektiven, Freiburg im Breisgau 1999.

Sozialarbeitsforschung: Was sie ist und leistet. Eine Bestandsaufnahme, hg. von Erika Steiner/Birgitta Sticher-Gil/Peter Sommerfeld/Konrad Maier, Freiburg im Breisgau 1998.

Thomas Rauschenbach/Werner Thole (Hg.): Sozialpädagogische Forschung. Gegenstand und Funktionen, Weinheim 1998.

Piotr Salustowicz/Bernd Horn/Norbert Klinkmann: Forschung an Fachhochschulen. Der Weg in eine neue Identität?, Weinheim 1992.

Maja Heiner (Hg.): Praxisforschung in der sozialen Arbeit, Freiburg im Breisgau 1988.
Assistenten ihren Lebensunterhalt mit Forschung verdient. Entscheidend für die Entwicklung einer guten Grundlagenforschung im Bereich Sozialer Arbeit ist deswegen, dass sich diese Wissenschaftlerinnen und Wissenschaftler auf die Fragestellungen der Sozialen Arbeit einlassen und die Soziale Arbeit zu ihrem Thema machen.

Ausgesprochen kontraproduktiv ist hierbei die Verankerung eines eigenständigen Fachs "Sozialarbeitswissenschaft « in den Fachhochschulstudiengängen Soziale Arbeit neben den »Bezugsdisziplinen «, insbesondere wenn damit die Forderung verbunden ist, dass diese Sozialarbeitswissenschaft nur von gelernten Sozialarbeiterinnen und Sozialarbeitern gelehrt werden dürfe. Damit werden die forschungserfahrenen Professorinnen und Professoren zurückverwiesen auf ihre Herkunftsdisziplin, in der Forschung in der Regel leichter zu finanzieren ist und vermutlich mehr Reputation bringt.

Die komplexen Fragestellungen der Sozialen Arbeit erfordern jedoch zum einen hochprofessionelle Forschung mit elaborierten Methoden der empirischen Sozialforschung und zum anderen das $\mathrm{Zu}$ sammenwirken von Vertretern verschiedener Herkunftsdisziplinen. So hat Jane Addams 1930 festgestellt: »... im Umgang mit sozialen Problemen stützen sich die Settlementhäuser einmal auf den Soziologen, dann auf den Psychiater; sie suchen die Dienste des Künstlers, Ökonomen, Turnlehrers, Caseworkers, Dramaturgen wie der ausgebildeten Krankenschwester; an einem Tag benötigen sie den Anthropologen, um einen Hinweis über die Eigenheiten einer neuen Immigrantengruppe zu erhalten ... selbst wenn der Vorwurf der Zersplitterung stimmen sollte, so antworten wir, dass wir noch immer viel weniger differenziert und komplex als das Leben selber sind. «Die Vorstellung, dass diese Komplexität von grundständig ausgebildeten »Sozialarbeitswissenschaftlerinnen " und »Sozialarbeitswissenschaftlern « unter der Chiffre »Transdisziplinarität « geleistet werden könnte erscheint geradezu grotesk. Interdisziplinäres Zusammenwirken ist hier gefordert.

Während die aktuelle Diskussion um die Professionalisierung der praktischen Sozialen Arbeit auf Abgrenzung von andern Berufen und Professionen zielt, muss es im Bereich von Wissenschaft und For- schung um die Integration ganz unterschiedlicher wissenschaftlicher Qualifikationen auf die Fragestellungen der Sozialen Arbeit gehen. Hierfür bieten die Fachhochschulgänge Soziale Arbeit geradezu ideale institutionelle Rahmenbedingungen und können eine entsprechende Kultur entwickeln, in der auch Sozialarbeiterinnen und Sozialarbeiter, Sozialpädagoginnen und Sozialpädagogen zu Forschung qualifiziert werden.

\section{Expertenwissen für Führungskräfte}

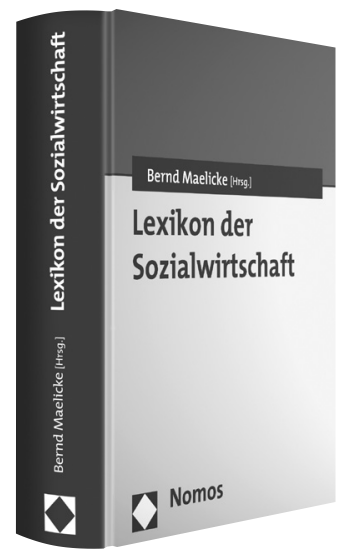

Lexikon der Sozialwirtschaft

Herausgegeben von

Prof. Dr. Bernd Maelicke

2008, 1.128 S., geb., 98,-€,

ISBN 978-3-8329-2511-6

Das Werk umfasst ca. 700 Stichworte von über 130 Autoren aus den einschlägigen Fachdisziplinen Betriebswirtschaft, Volkswirtschaft, Sozialpolitik, New Public Management, Recht, Erziehungswissenschaften, Sozialarbeit/Sozialpädagogik und Psychologie und richtet sich an die Führungskräfte in der Sozialwirtschaft/Sozialmanagement, der Aus- und Weiterbildung sowie an die Wissenschaft.

\section{Nomos}

Bitte bestellen Sie im Buchhandel oder versandkostenfrei unter $\mathbf{w w w}$.nomos-shop.de 